\title{
Control of VR-7 Dynamic Stall by Strong Steady Blowing
}

\author{
D. Weaver* \\ Lockheed Martin Missiles and Fire Control, Dallas, Texas 75265-003 \\ K. W. McAlister ${ }^{\dagger}$ \\ NASA Ames Research Center, Moffet Field, California 94035-1000 \\ and \\ J. $\mathrm{Tso}^{\ddagger}$ \\ California Polytechnic State University, San Luis Obispo, California 93407
}

\begin{abstract}
An experiment was performed in a water tunnel on a Boeing-Vertol VR-7 airfoil to study the effects of tangential blowing over the upper surface. Blowing was applied at the quarter-chord location during sinusoidal pitching oscillations described by $\alpha=\alpha_{m}+10 \mathrm{deg} \sin \omega t$. Results were obtained for a Reynolds number of $1 \times 10^{5}$, mean angles of 10 and $15 \mathrm{deg}$, reduced frequencies ranging from 0.005 to 0.15 , and blowing rates from $C_{\mu}=0.16$ to 0.66 . Unsteady lift, drag, and pitching moment loads are reported, along with fluorescent-dye flow visualizations. Strong steady blowing was found to prevent the bursting of the leading-edge separation bubble at several test points. When this occurred, the lift was increased significantly, stall was averted, and the shape of the moment response showed a positive damping in pitch. In almost all cases, steady blowing reduced the hysteresis amplitudes present in the loads, but the benefits diminished as the reduced frequency and mean angle of oscillation increased. A limited number of pulsed blowing cases indicated that for low blowing rates, the greatest gains were achieved at $\boldsymbol{F}^{+}=0.9$.
\end{abstract}

\section{Nomenclature}

$b \quad=$ model span

$C_{d}=$ drag coefficient, $\operatorname{drag} /\left(q_{\infty} S\right)$

$C_{l}=$ lift coefficient, lift $/\left(\boldsymbol{q}_{\infty} \boldsymbol{S}\right)$

$C_{m}=$ moment coefficient, moment $/\left(\boldsymbol{q}_{\infty} \boldsymbol{S} \boldsymbol{c}\right)$

$C_{\mu}=$ mean blowing coefficient, $J / \boldsymbol{q}_{\infty} \boldsymbol{c}, 2(\boldsymbol{h} / \boldsymbol{c})\left(\boldsymbol{V}_{s} / \boldsymbol{V}_{\infty}\right)^{2}$

$c=$ model chord

$F^{+}=$dimensionless pulsing frequency, $f x_{\text {te }} / V_{\infty}$

$f \quad=$ pulsing frequency

$h=$ slot height

$J=$ mean momentum at slot exit (per unit span), $\rho \boldsymbol{V}_{s}^{2} \boldsymbol{h}$

$k=$ reduced frequency, $\boldsymbol{\omega} \boldsymbol{c} / 2 \boldsymbol{V}_{\boldsymbol{x}}$

$Q_{s}=$ volume-flow rate through slot

$q_{\infty}=$ dynamic pressure, $\rho \times V_{\infty}^{2} / 2$

$R e=$ Reynolds number, $c V_{x} / \nu$

$S=$ planform area of airfoil, $\boldsymbol{b c}$

$T=$ period between flow pulses

$t=$ time

$V_{s}=$ mean slot-exit velocity

$V_{\infty}=$ freestream velocity

$x=$ distance along chord from leading edge

$x_{\mathrm{te}}=$ distance from slot to trailing edge

$\alpha=$ angle of attack

$\alpha_{a}=$ amplitude of airfoil oscillation

$\alpha_{m}=$ mean angle of airfoil oscillation

$\delta=$ duty cycle, $\tau / T$

$\zeta \quad=$ pitch damping, (closed integration)

$$
-\int C_{m} \frac{\mathrm{d} \alpha}{4 \alpha_{a}^{2}}
$$

$v \quad=$ fluid kinematic viscosity

Presented as Paper 98-2413 at the 16th Applied Aerodynamics Conference-Unsteady Aerodynamics Session, Albuquerque, NM, 15 June 1998; received 6 August 2003; revision received 29 January 2004; accepted for publication 6 February 2004. This material is declared a work of the U.S Government and is not subject to copyright protection in the United States. Copies of this paper may be made for personal or internal use, on condition that the copier pay the $\$ 10.00$ per-copy fee to the Copyright Clearance Center, Inc., 222 Rosewood Drive, Danvers, MA 01923; include the code $0021-8669 / 04 \$ 10.00$ in correspondence with the CCC.

${ }^{*}$ Research Engineer Senior, Aerodynamics.

${ }^{\dagger}$ Research Scientist, Aeroflightdynamics Directorate.

$\ddagger$ Professor, Aerospace Engineering. Associate Fellow AIAA. $\rho \quad=$ fluid density
$\tau \quad=$ duration of a single flow pulse
$\omega \quad=$ frequency of pitch oscillation

\section{Introduction}

A SIGNIFICANT limitation on helicopter performance is a phenomenon called retreating-blade stall. ${ }^{1}$ Although stall can occur on any wing when the angle of attack is too high, this condition is especially damaging on a helicopter rotor in forward flight because of the large impulsive loads that are imparted to each blade and then transmitted to the hub. ${ }^{2}$ These unsteady loads can result in severe vibrations that are not only destructive to the rotor system and airframe, but can have a deleterious affect on the crew. ${ }^{3}$ Therefore, it is important to investigate any practical means of attaining higher blade angles of attack without incurring stall.

\section{Dynamic Stall Description and Experiment Goal} Dynamic Stall

Dynamic stall is a process in which the boundary layer rapidly separates from an airfoil or wing while it is undergoing a dynamic increase in angle of attack. ${ }^{4.5}$ In the case of retreating-blade stall, the increase in angle of attack is due to the sinusoidal-pitching motion that is experienced by the helicopter rotor blades. Because the rotational effects on the rotor flowfield are considered to be small, ${ }^{1,4-6}$ dynamic stall studies are often carried out in simple, two-dimensional experiments. Additionally, because rotor blades typically have a high aspect ratio, tip effects are also of secondary importance. Experiments dealing with dynamic stall are usually conducted on oscillating airfoils with a sinusoidal-pitching motion defined by

$$
\alpha=\alpha_{m}+\alpha_{a} \sin \omega t
$$

The differences between dynamic stall and quasi-steady stall stem from the unsteady motion of the airfoil, as given by $\mathrm{d} \alpha / \mathrm{d} t$. Three prominent differences can be identified. ${ }^{1.4 .5}$ The first difference is the appearance of a large amount of load hysteresis with respect to angle of attack in the unsteady case. Because the airfoil is oscillating, the relative flow velocity experienced by the airfoil during the upstroke portion of the cycle is different from the downstroke. In particular, the boundary layer is attached for nearly all of the upstroke, but it is separated for most of the downstroke. 
The second difference is a phenomenon called lift overshoot. Past experiments show that a pitching airfoil tolerates large regions of reversed flow on its surface before succumbing to large-scale, boundary-layer separation. ${ }^{4}$ This tolerance allows the airfoil to rotate well beyond the quasi-steady stall angle and attain a much higher lift force than would normally occur.

The third difference between dynamic stall and quasi-steady stall is the shedding of a concentrated vortex from the upper surface (also reported by Ham, ${ }^{6}$ Van Dommelen and Shen, ${ }^{7}$ Choudhuri et al., Shih et al., ${ }^{9}$ and Walker ${ }^{10}$ ). The concentrated vorticity that is generated and then shed from the airfoil is commonly referred to as the dynamic stall vortex. This shedding event, which normally occurs during the upstroke, causes impulsive changes in the loads on the airfoil and is accompanied by large-scale, boundary-layer separation.

Parameters that Influence Dynamic Stall

Many factors have been found to influence the dynamic stall process. Light stall, characterized by a relatively weak stall vortex and a viscous zone on the order of the airfoil thickness, is found to be sensitive to the same geometrical parameters that affect quasi-steady stall. ${ }^{1,4,5}$ Leading-edge radius and airfoil camber are examples of geometric parameters that can affect light stall. Light stall typically occurs when the oscillation rate is low and the amplitude does not carry the airfoil very much beyond the quasi-steady stall angle.

Deep stall, on the other hand, is relatively unaffected by airfoil geometry. Deep stall exhibits large-scale vortex shedding as its predominant feature (Fig. 1). Large force overshoots and hysteresis amplitudes are present, and the scale of the viscous zone is on the order of the airfoil chord. Deep stall is heavily influenced by the amount of time the airfoil spends above the quasi-steady stall angle and the maximum angle that the airfoil eventually reaches.

\section{Control of Dynamic Stall}

The discovery that a simple change in the shape of the profile has little or no affect on the nature of deep dynamic stall has motivated many studies to focus on more unconventional ways to control
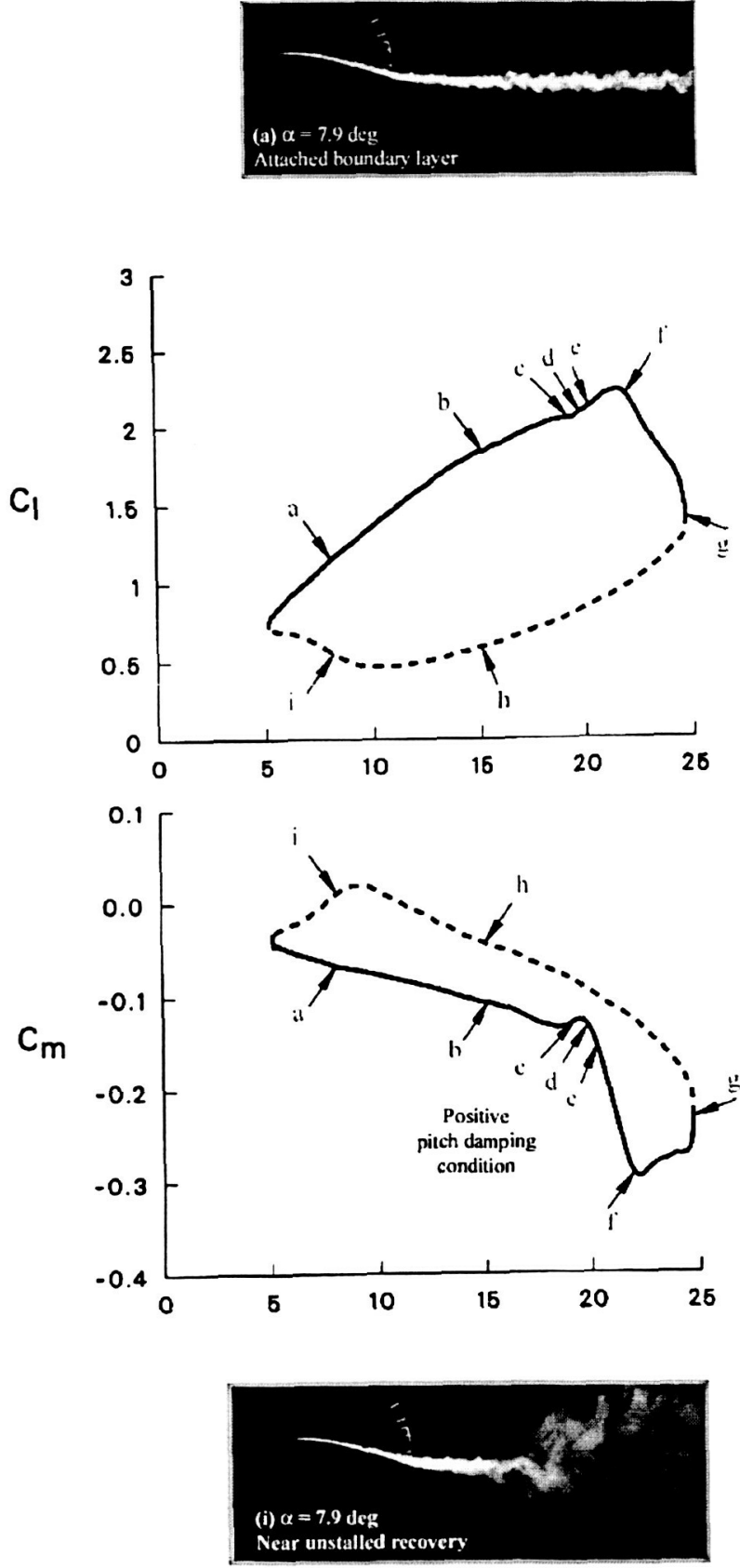
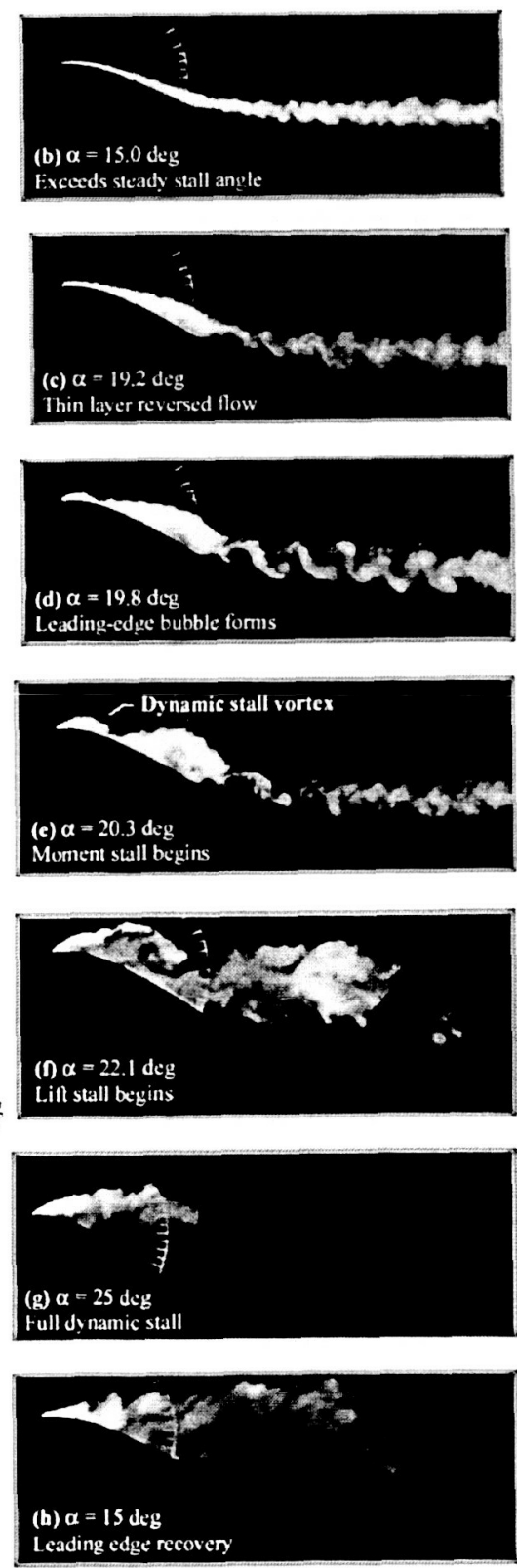

Fig. 1 Dynamic stall events on the VR-7 airfoil for $\alpha=15+10 \mathrm{deg} \sin (\omega t)$ with $k=0.1$ and $\operatorname{Re}=2 \times 10^{5}$ (McAlister and Tung ${ }^{11}$ ). 


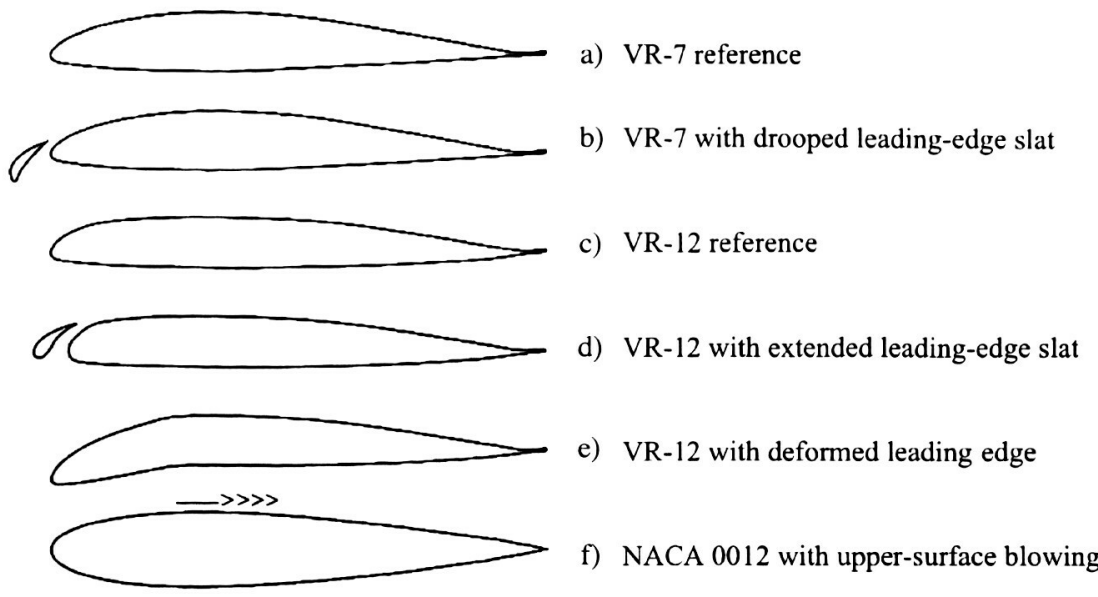

Fig. 2 Airfoil configurations studied in AFDD water tunnel facility for controlling dynamic stall.
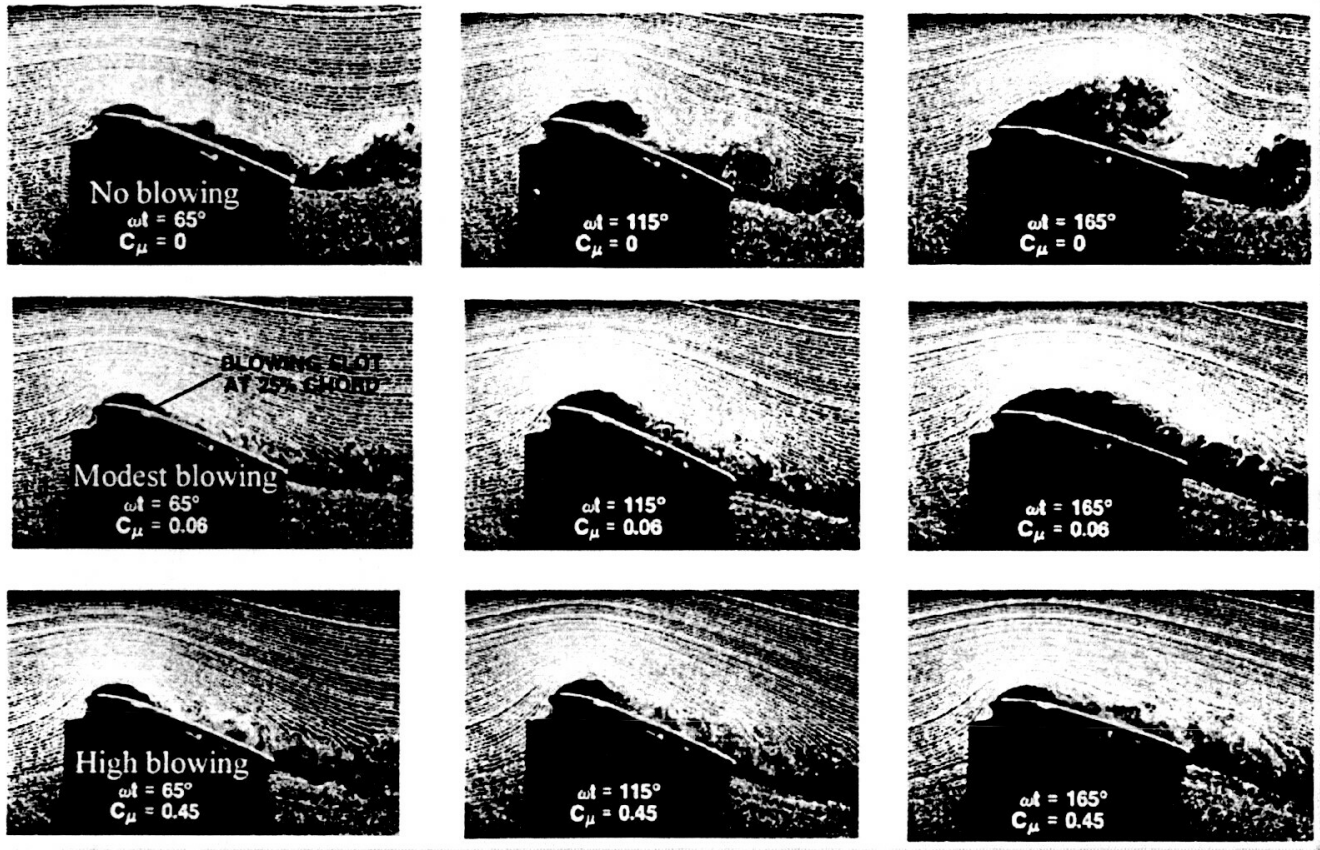

Fig. 3 Blowing effects on dynamic stall occurring on a NACA 0012 airfoil undergoing $\alpha=10+10 \mathrm{deg} \sin \omega t$ at $k=0.5$ and $R e=3 \times 10^{4}$ (unpublished study by McAlister and Carr).

this event. A brief summary of previous work, including configurations (Fig. 2) explored at the U.S. Army Aeroflightdynamics Directorate (AFDD), located at NASA Ames Research Center, is provided next.

\section{Slatted Airfoils}

In this novel approach, Boeing-Vertol VR-7 (McAlister and Tung $^{11}$ ) and VR-12 (Plantin de Hugues et al. ${ }^{12}$ ) airfoils were fitted with different styles of leading-edge slats and tested in a closedcircuit water tunnel. The best results were obtained with the VR-7 airfoil fitted with an optimally positioned NACA 15320 slat. The slat eliminated the formation of the dynamic stall vortex for reduced frequencies up to 0.2 and for oscillations as high as $20+10 \mathrm{deg} \sin$ $\omega t$. Hysteresis amplitudes, as well as the peak values for drag and pitching moment, were also dramatically decreased. The only negative effect observed was an increase in the drag coefficient at low angles of attack.

\section{Deformed Airfoils}

Another effective attempt at controlling dynamic stall involved the use of a deformed-leading edge airfoil. ${ }^{13}$ In this study, the for- ward $25 \%$ of a VR-12 airfoil was drooped by $13 \mathrm{deg}$. Test results from the water tunnel showed that the dynamic stall vortex had been averted and that the hysteresis amplitudes were significantly reduced. The maximum pitching moment and drag coefficients were much lower than those of the unmodified airfoil. As with the airfoils with slats, the deformed airfoil experienced a drag penalty at lower angles of attack because of the profile change.

A numerical study of a dynamically deformed leading-edge configuration was conducted on a NACA 0012 airfoil at a freestream Mach number of 0.3 (Sahin et al. ${ }^{14}$ ). The results indicated that it is the shape itself, not the time rate of change of the deformation that is responsible for the improved performance. A related shapeadaptation concept has also been employed to successfully control dynamic stall under compressible flow conditions. ${ }^{15}$

\section{Steady Upper-Surface Blowing}

In addition to studies involving actual changes in the shape of the airfoil, an unpublished flow-visualization study of steady uppersurface blowing was conducted by McAlister and Carr (Fig. 3) at the AFDD during the early 1980s (Yu et al. ${ }^{16}$ ). In this water-tunnel study, a NACA 0012 airfoil was constructed with a blowing slot on 
the quarter-chord line of the upper surface. Pressurized water was injected into the boundary layer from the slot to help overcome the adverse pressure gradient. The results demonstrated that blowing was capable of trapping the dynamic stall vortex near the leading edge. The visualization photographs also presented clear evidence that the highest blowing rate was most effective. This was confirmed in a later study where the unsteady loads were measured, the precedent to this paper (Weaver et al. ${ }^{17}$ ).

Steady blowing at the leading edge and flap shoulder of a multielement airfoil has also been studied at Tel Aviv University (Greenblatt and Wygnanski ${ }^{18,19}$ ). Their results have shown that blowing at the leading edge of a NACA 0015 airfoil actually has a detrimental effect by enlarging the hysteresis loops of the lift, drag, and moment loads for $C_{\mu}<1 \%$.

\section{Periodic Excitation}

In the same series of studies at Tel Aviv University, periodic blowing with zero-mass flux was imposed on a NACA 0015 airfoil undergoing pitch oscillations under incompressible conditions at rotorcraft reduced frequencies. ${ }^{18,19}$. The flow was excited at the leading edge and flap shoulder to maximize airfoil performance while limiting the moment excursions to a prestall value. Incidence angle excursions were confined to $\pm 5 \mathrm{deg}$. Reduced excitation frequencies ranged from $\boldsymbol{F}^{+}=0.3$ to 5 , excitation amplitudes from $C_{\mu}=0.01$ to 5, and Reynolds numbers from $0.3 \times 10^{6}$ to $0.9 \times 10^{6}$. The studies concluded that for various flap deflections, leading-edge oscillatory excitation is far superior to steady blowing due to the latter's undesirable enlargement of the hysteresis loops. It was further concluded that flap-shoulder excitation is superior to leadingedge excitation, whose effectiveness is more erratic in its dependence on $\boldsymbol{F}^{+}$and $\boldsymbol{C}_{\boldsymbol{\mu}}$. For the same $\boldsymbol{C}_{l, \max }$, flap-shoulder excitation also has about one-third of the mean form drag of its leading-edge counterpart.

Dynamic stall control using zero-mass flux periodic excitation has also been numerically investigated. ${ }^{20}$ This study involved a NACA 0015 airfoil undergoing $\alpha=13+5 \mathrm{deg} \sin \omega t$ at a reduced frequency of 0.05 . The calculations were carried out for both incompressible and compressible flows. The effects of control parameters, such as excitation location, exit angle, momentum coefficient $\left(\boldsymbol{C}_{\mu}=0.5 \%\right.$ and $\left.2.5 \%\right)$, and pulsating frequency $\left(\boldsymbol{F}^{+}=3.1\right.$ and 6.3 ), were examined. The periodic excitation was found to be effective in suppressing dynamic stail in both incompressibie and conpressible flows. The most effective excitation location was found to be at $70 \%$ of the chord. Excitation at the $10 \%$ chord location was found to cause significant load oscillations at the excitation frequency.

\section{Goal of the Current Experiment}

The goal of this study was to build on the informal study conducted at the AFDD in the early 1980s, this time employing both flow visualization and load measurements to evaluate the effects of steady blowing when applied at the quarter-chord of a VR-7 airfoil. The sinusoidal-pitching motion (in degrees) of the airfoil was fixed at

$$
\alpha=\alpha_{m}+10 \sin \omega t
$$

Measurements were made at a Reynolds number of $1 \times 10^{5}$. Flow-visualization photographs were taken during each cycle of oscillation to complement the force and moment data.

\section{Test Description}

\section{Water-Tunnel Facility}

The test was conducted in the AFDD closed-circuit water tunnel at NASA Ames Research Center. The test section measures $34 \mathrm{in.}(864 \mathrm{~mm})$ long (chordwise direction), $8.3 \mathrm{in} .(211 \mathrm{~mm})$ wide (spanwise direction), and 12 in. (305 mm) high. ${ }^{11}$ Two-dimensional airfoils are supported on both sides of the test section by load cells in the lift and drag directions. Torsionally stiff bellows and lip seals provide water containment. A honeycomb and several screens are positioned upstream of the test section in the settling chamber to straighten and smooth the flow. The turbulence intensity in the test section was found to be about $0.05 \%$ when there is no model present. With a typical model installed in the test section, the turbulence intensity can increase by a factor of five.

\section{Airfoil Model}

The model used for the test was a Boeing-Vertol VR-7 airfoil with a negative 3-deg trailing-edge tab (an upward deflection of the trailing edge). A profile of the airfoil is shown in Fig. 2. The model has a chord length of 4 in. and a span of nearly 8.3 in. (allowing for minimal clearance with the test-section walls).

The blowing slot was defined by a contoured passage, formed by two upper-surface sections with an exit gap of 0.003 in. located at the quarter chord of the airfoil. Several diffuser passages were cut into the interior of the upper-rear piece to guide the flow evenly to the slot. Each passage has a similar area ratio, and they all fan out from a central plenum that is fed from the port side of the model through a channel inside the spar. The spar is coincident with the quarter-chord axis of the airfoil.

To perform the flow visualizations, two dye ports were installed along the midspan of the model. One port was located at the leading edge and the other was located inside the slot. The ports were fed by separate passages that ran through the interior of the model. The passages were connected to separate tubes that entered the model from the starboard side.

\section{Blowing System}

Flow to the slot on the upper surface of the model was carried through a flexible tube that was connected to two pumps, operating in series to pull water from the tunnel. This arrangement provided water to the slot with the same temperature as the freestream flow. The use of two pumps was required to supply sufficient pressure for a wide range of blowing momentum coefficient $\boldsymbol{C}_{\boldsymbol{\mu}}$. A globe valve and a venturi-style flow meter were located between the two pumps to control accurately and monitor the volume-flow rate through the slot.

The slot momentum coefficient, a parameter that states the relative strength of the blowing, is defined by

$$
C_{\mu}=J / q_{\infty} c=2(h / c)\left(V_{s} / V_{\infty}\right)
$$

Physicaliy, this coefficient represents the ratio of slot momentum to freestream momentum.

A limited number of pulsed-blowing cases were considered. To perform pulsed blowing, a rotary valve was placed in the line between the airfoil and pump 2. Water entered a holding chamber, and pulses of water were released when holes in a rotating plate became aligned with an exit hole in the valve body. In other words, the valve caused the mass-flow rate to oscillate between zero and some maximum value and is, therefore, different (and certainly less efficient) than a zero-mass flow system. The rotational speed of the motor was adjusted with an electronic speed controller. There was no particular phase relationship between the frequency of the blowing pulses, $f$, and the frequency of airfoil oscillation, $\omega / 2 \pi$ because $f \gg \omega / 2 \pi$. A nondimensional value for the pulsing frequency is given by

$$
F^{+}=f x_{\mathrm{te}} / V_{\infty}
$$

The meter used in this experiment gave a mean value for the volume flow rate. To compare with tests in which the rms value is given, an assumption must be made about the waveform of the jet flow. Let

$$
\begin{aligned}
& f(t)=A \sin (\pi t / \tau) 0 \leq t \leq \tau \\
& f(t)=0 \quad \tau<t \leq T
\end{aligned}
$$

where $\tau$ is the duration of the flow and $T$ is the period between pulses. Then,

$$
\mathrm{rms} / \text { mean }=\left(\pi^{2} / 8 \delta\right)^{\frac{1}{2}}
$$


where $\delta$ is the duty cycle and is equal to the fraction of time that flow is passing through the valve. If $\delta=0.5$, for example, then $\mathrm{rms} /$ mean $=1.57$.

\section{Airfoil Installation and Instrumentation}

In addition to the suspension system for measuring the unsteady hydrodynamic loads, a transducer was mounted on the axis of rotation to measure the airfoil's incidence. A pressure transducer, connected between the settling chamber and the test section, was used to measure the dynamic pressure, from which the freestream velocity $V_{\infty}$ was calculated. During the pulsed-blowing portion of the experiment, a strobe was used to monitor the rotational speed of the pulse valve. For this part of experiment, rather than using the rms slot velocity to compute $\boldsymbol{C}_{\mu}$, the time-averaged velocity deduced from the flow meter was used.

Calibrations based on the application of known loads on the force transducers, strain-gauge bridges, incidence transducer, and pressure transducer were found to be linear within the ranges of interest. The estimated measurement accuracy for angle of attack is $0.2 \mathrm{deg}$, for lift and drag is $0.002 \mathrm{lb}$, and for the pitching moment is $0.02 \mathrm{in} .-\mathrm{lb}$.

\section{Data Acquisition and Processing}

Each of the signals from the measurement devices was amplified, digitized, recorded, and processed by a remote data-acquisition system. The acquisition system was a stand-alone unit consisting of amplifiers, an analog-to-digital converter, and a digital workstation. A program instructed the acquisition system to digitize the data signals based on a 360 per-rev pulse train that was synchronous with $\omega t$, and a $1 /$ revolution pulse that was synchronous with the beginning of each cycle. The program then ensemble averaged the digitized cycles. Data averaging was performed over a period of 30 cycles for the unsteady data and over a period of 5 cycles for the quasi-steady data.

Dynamic load tares were frequently recorded throughout the experiment to provide current reference (or zero) levels for all of the transducers. These were obtained by recording data at a static, noflow condition (model set at the mean angle of attack with $\boldsymbol{q}_{\infty}=0$ ) and at a dynamic, no-flow condition (model oscillating slowly with $\boldsymbol{q}_{\infty}=0$ ). Following the averaging operation, each signal was appropriatcly adjusted using either the static or dynamic zero reference. All of the force and moment data were reduced to non-dimensional coefficients based on the instantaneous value for the freestream dynamic pressure $q_{\infty}(t)$. It was necessary to use the instantaneous dynamic pressure because the oscillating airfoil produced a significant variation in tunnel resistance (or blockage) and, hence, a varying $q_{\infty}$ throughout the cycle.

No corrections were applied to the data to account for inertialpitching moments or to account for forces caused by the injection of water into the airfoil. Corrections were not used for inertial pitching moments because an estimate showed that they were approximately two orders of magnitude less than the fluid-dynamic moments. Corrections were not needed to account for forces associated with the supply of water for blowing because the water was introduced along the axis of the spar, normal to the lift-drag plane. Additionally, no corrections were made that account for the presence of the tunnel walls.

\section{Flow Visualization}

Flow visualization was performed to help interpret the results of the load measurements. Fluorescent dye was released from two ports located midspan on the model. The visualization was performed with the force balance removed from the viewing side of the tunnel. This provided an unobstructed view along the axis of the model's spar. Single-frame recordings were made during the oscillation cycles by dimming the lights, opening the shutter of the camera, and firing the strobe lamp. An electronic monitoring circuit was used to flash the strobe at selectable values of $\omega t$. Light-emitting diodes were positioned in the field of view of the camera to display the digital values of $\omega t$
The dye used for visualizing the flow was made by mixing a rhodamine $6 \mathrm{G}$ concentrate with deionized water. This dye has an orange color in ordinary room lighting, but it provides a fluorescentyellow emission when stimulated by light of a shorter wavelength. The dye was illuminated by a xenon strobe that emitted a large quantity of radiation in the ultraviolet range. The strobe cavity had the shape of a long tube so that light from the strobe needed only to be baffled to form a sheet of light with a thickness of about 1 in. The light passed through the upper test-section window and straight down onto the airfoil surface. The photographs were taken with a Hasselblad camera loaded with Kodak TMY 6053 black-and-white film (ASA 400).

\section{Blowing Results}

Unsteady loads were measured on the airfoil as it oscillated about its quarter-chord axis at several mean angles and reduced frequencies. The oscillation amplitude in this experiment was fixed at $10 \mathrm{deg}$. Blowing-momentum coefficients were chosen to compare with previous experimental studies. ${ }^{7}$ To match the higher values of $C_{\mu}$, it was necessary to limit the freestream Reynolds number to $1 \times 10^{5}$. Testing at a higher Reynolds number could have been performed with a stronger blowing system, but an earlier experiment demonstrated that the unsteady-load coefficients on a VR-7 do not vary appreciably over the range of Reynolds numbers that can be achieved in this facility. ${ }^{11}$

Steady-state loads were not directly measured in this experiment. Tests have shown that loads measured at very low reduced frequencies (quasi-steady loads) are approximately the same as true steady-state loads. ${ }^{12}$ Although every effort was made to record data at nominal conditions, some variation in Reynolds number $R e$, reduced frequency $\boldsymbol{k}$, and momentum coefficient $\boldsymbol{C}_{\mu}$, was observed. This variation is the result of using the mean dynamic pressure to compute $V_{\infty}$, which appears in each of the cited coefficients. This quantity varies from cycle to cycle because of the unsteady testsection blockage introduced by the airfoil's motion.

Flow-visualization photographs were obtained at several points in each cycle. The photographs for all quasi-steady oscillations are sequenced according to $\alpha$ instead of $\omega t$ because the flowfield is not appreciably different from a true steady-state condition. Photographs taken at higher reduced frequencies are sequenced according to values of $\omega t$. The correspondence between $\alpha$ and a particular value of $\omega t$ depends on the mean angle of attack and is governed by $\alpha=\alpha_{m}+10$ deg $\sin \omega t$. Steady blowing test conditions are summarized in Table. 1

\section{Steady Blowing with $\alpha_{m}=10$ Degrees and $k=0.005$ (Nominal)}

Increasing the blowing rate produces progressively higher liftcurve slopes (Fig. 4), rising by $23 \%$ for $\boldsymbol{C}_{\mu}=0.16$ and by $30 \%$ for $C_{\mu}=0.54$. Separation is delayed by about $5 \mathrm{deg}$ and the maximumlift coefficient is increased by $33 \%$ for $\boldsymbol{C}_{\mu}=0.16$. Blowing at $C_{\mu}=0.54$ prevents separation and increases the maximum-lift coefficient by $67 \%$. Both blowing rates eliminate the small lift spike that appears near $\alpha=16 \mathrm{deg}$ without blowing. Lift hysteresis is essentially eliminated for both blowing rates.

Table 1 Steady-blowing test conditions

\begin{tabular}{lcclc}
\hline \hline $\boldsymbol{\alpha}_{m}$, deg & $\boldsymbol{k}$ & $\boldsymbol{Q}_{s}, \mathrm{gal} / \mathrm{min}$ & \multicolumn{1}{c}{$\boldsymbol{C}_{\mu}$} & $\boldsymbol{V}_{s} / \boldsymbol{V}_{\infty}$ \\
\hline 10 & 0.004 & 0.0 & 0.0 & 0.0 \\
10 & 0.005 & 10.2 & 0.16 & 1.3 \\
10 & 0.006 & 18.5 & 0.54 & 19.0 \\
10 & 0.05 & 0.0 & 0.0 & 0.0 \\
10 & 0.05 & 10.2 & 0.16 & 10.3 \\
10 & 0.05 & 18.5 & 0.57 & 19.5 \\
10 & 0.15 & 0.0 & 0.0 & 0.0 \\
10 & 0.15 & 10.2 & 0.16 & 10.3 \\
10 & 0.15 & 18.5 & 0.56 & 19.3 \\
15 & 0.05 & 0.0 & 0.0 & 0.0 \\
15 & 0.05 & 10.2 & 0.19 & 11.3 \\
15 & 0.05 & 18.5 & 0.66 & 21.0 \\
15 & 0.15 & 0.0 & 0.0 & 30 \\
15 & 0.15 & 18.5 & 0.65 & 30 \\
\hline
\end{tabular}



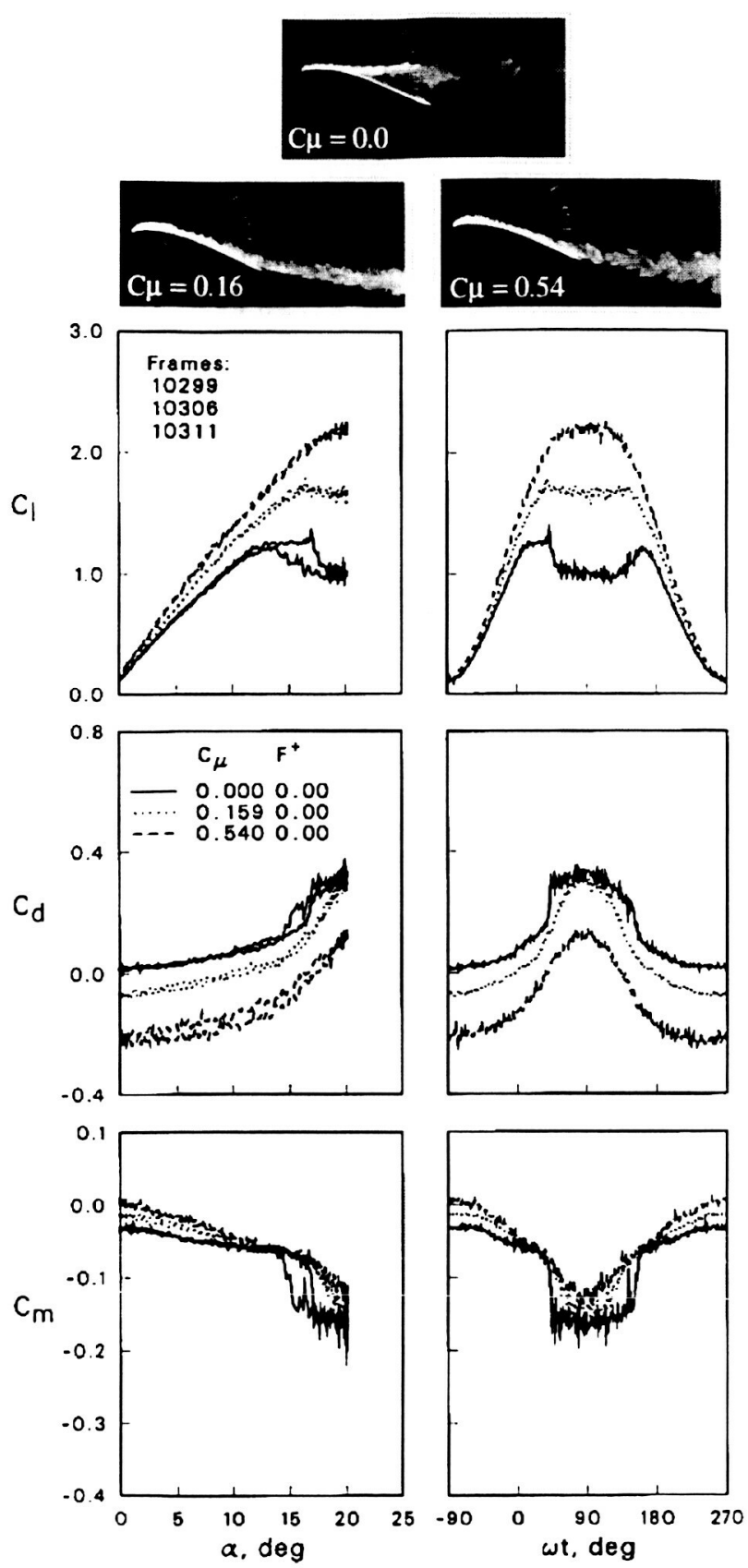

Fig. 4 Flow visualization ( $\alpha=15 \mathrm{deg}$ and $\omega t=30 \mathrm{deg}$ ) and load measurements for $\alpha_{m}=10 \mathrm{deg}$ and $k=0.005$ (nominal) with different steady blowing rates.

Blowing has a noticeable effect on drag, driving it toward large negative values due to jet thrust. Blowing at $\boldsymbol{C}_{\mu}=0.16$ produces a roughly uniform downward shift of 0.09 , which results in a negative drag coefficient for half of the cycle. Blowing at $\boldsymbol{C}_{\mu}=0.54$ lowers $C_{d}$ by roughly 0.23 , so that the drag is negative for all but $2.5 \mathrm{deg}$ of incidence during the cycle. This drag reduction is an order of magnitude larger than those reported with periodic excitation. ${ }^{19,20}$ The jet thrust also adds minor gains in the slope of the lift curve and maximum $C_{l}$. These gains would not have been realized by blowing at the leading-edge location without having a detrimental affect on the pitching moment. Although a small amount of hysteresis is present, the sharp drag increase at the onset of stall does not appear for either of the blowing cases.

Blowing produces some interesting changes in the quarter-chord moment. The positive shift that occurs at $\alpha=0$ deg is probably caused by an increase in the velocity of the flow over the leading edge. A high-speed jet tends to drag the surrounding flow with it through viscous interaction. Part of the surrounding flow in this situation is made up of fluid that previously formed the leadingedge boundary layer. The end result is more leading-edge suction. Because the pressure is lowered in front of the reference axis for the pitching moment, the moment coefficient becomes more positive. Another interesting change that occurs with increasing blowing rate is the progressive increase in the magnitude of the pitching-moment slope for $\alpha<13 \mathrm{deg}$ and a decrease in the magnitude of the pitching moment at high angles of attack. Moment hysteresis is reduced to negligible levels at both blowing rates.

Detailed flow visualizations ${ }^{17}$ show that, without blowing, there is a progressive increase in the boundary-layer thickness between $\alpha=5$ and $12 \mathrm{deg}$, after which the separation point steadily moves forward from the trailing edge, reaching the leading edge at an angle of attack near $16 \mathrm{deg}$. When blowing at $\boldsymbol{C}_{\mu}=0.16$ is applied to the quasi-steady oscillation, a separation bubble near $x / c=0.13$ develops at $\alpha=15 \mathrm{deg}$. At higher angles of attack, the boundary layer over the remainder of the airfoil becomes very thick. At $\alpha=17 \mathrm{deg}$, the boundary layer appears to be highly turbulent over most of the surface, and at $\alpha=18$ deg a substantial region of trailing-edge separation is present. Blowing at $C_{\mu}=0.54$ prevents massive separation from occurring on the airfoil. A separation bubble, initially visible at $\alpha=15 \mathrm{deg}$, still forms at this high blowing rate. At higher angles of attack, the size of the separation bubble increases and the boundary layer becomes very thick. Large, turbulent structures are visible in the boundary layer at the maximum angle of attack $(\alpha=20 \mathrm{deg})$. No trailing-edge separation is visible in this case.

\section{Steady Blowing with $\alpha_{m}=10$ Degrees and $k=0.05$}

Stall is progressively delayed as the blowing rate is increased (Fig. 5). Blowing at both rates removes the distortion (the flat zone followed by a surge) in the lift curve and substantially extends the linear region before stall. The lift-curve slope during the upstroke increases by $15 \%$ for $C_{\mu}=0.16$ and by $25 \%$ for $C_{\mu}=0.57$, and the maximum-lift coefficient increases by 38 and $59 \%$, respectively. The magnitude of the downward shift in drag is similar to that for the quasi-steady case. As the blowing rate is increased, the moment coefficients increase (become more positive) at low angles of attack. In addition, increases in the blowing rate causes the magnitude of the moment-curve slope to increase progressively and the negative peak in the moment coefficient to decrease. In fact, the peak moment is reduced by $40 \%$ for $C_{\mu}=0.57$. Without blowing, a portion of the moment curve that lies between $\alpha=15$ and $18 \mathrm{deg}$ has a clockwise orientation, which represents negative-pitch damping during this portion of the cycle. This is an unstable region where energy is being extracted from the flow ${ }^{1}$ that is not present in either of the blowing cases.

Flow visualizations for the zero-blowing case ${ }^{17}$ indicate a rapid development of reversed flow as the airfoil rotates from $\omega t=20$ to $30 \mathrm{deg}$. At $\omega t=40 \mathrm{deg}$, a dynamic stall vortex is visible over the quarter-chord point while various boundary-layer eruptions are coalescing over the aft portion of the airfoil. At $\omega t=45 \mathrm{deg}$ the dynamic stall vortex has moved to about $\boldsymbol{x} / \boldsymbol{c}=0.5$ and a large structure has formed over the trailing edge. By the time the airfoil reaches $\omega t=50 \mathrm{deg}$, the dynamic stall vortex has been shed from the trailing edge, and the boundary layer has separated from the leading edge. When $C_{\mu}=0.16$, a separation bubble begins to form over the $x / c=0.125$ point at $\omega t=30 \mathrm{deg}$. At $\omega t=45 \mathrm{deg}$, large turbulent structures appear in the boundary layer as the jet begins to separate from the surface. At $\omega t=60 \mathrm{deg}$, the jet separates from the surface and the leading-edge bubble fails to close. For the highest blowing rate of $\boldsymbol{C}_{\mu}=0.57$, trailing-edge separation appears to be restrained during the cycle. The bubble reaches its maximum size near $\omega t=70 \mathrm{deg}$, after which it fails to close. At this point, the boundary layer is also very thick and contains large turbulent structures. No vortexlike structures are noticeable on the surface after the bubble is compromised. The jet appears to be slightly separated from the surface at $\omega t=90 \mathrm{deg}$. As the angle of attack decreases, the separation bubble re-forms and is no longer visible at $\omega t=180 \mathrm{deg}$. 

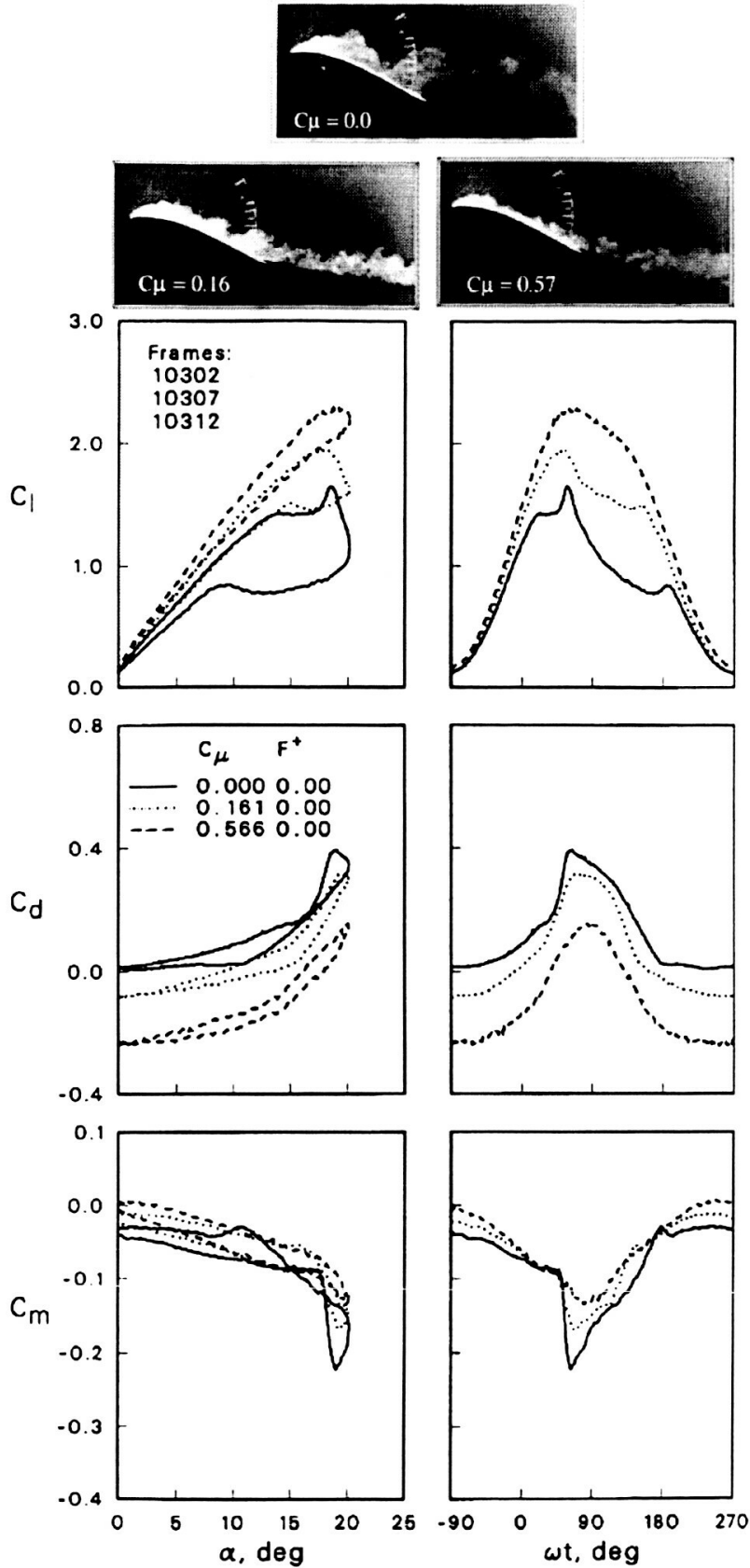

Fig. 5 Flow visualization (at $\alpha=17 \mathrm{deg}$ and $\omega t=45 \mathrm{deg}$ ) and load measurements for $\alpha_{m}=10 \mathrm{deg}$ and $k=0.05$ with different steady blowing rates.

Steady Blowing with $\alpha_{m}=10$ Degrees and $k=0.15$

The changes produced by blowing (Fig. 6) are similar to those observed at $\boldsymbol{k}=0.005$ and $\boldsymbol{k}=0.05$. Increasing the blowing rate progressively reduces the lift hysteresis. The lift-curve slope during the upstroke increases by $16 \%$ for $\boldsymbol{C}_{\mu}=0.16$ and $21 \%$ for $\boldsymbol{C}_{\mu}=0.56$. Increasing the momentum coefficient progressively delays the onset of stall. The maximum-lift coefficient increases by $19 \%$ for $C_{\mu}=0.16$ and $31 \%$ for $C_{\mu}=0.56$. The distortion in the lift curve before stall disappears at both blowing rates

Changes to the drag and pitching moment are also similar to those obtained at the lower reduced frequencies. A blowing thrust effect is evident in the drag curve, and the magnitude of the change appears to be about the same as that seen earlier. The moment coefficient increases at $\alpha=0 \mathrm{deg}$, and the magnitude of the slope becomes more negative with stronger blowing. The maximum negative moment decreases by $39 \%$ for $\boldsymbol{C}_{\mu}=0.56$ (essentially eliminating
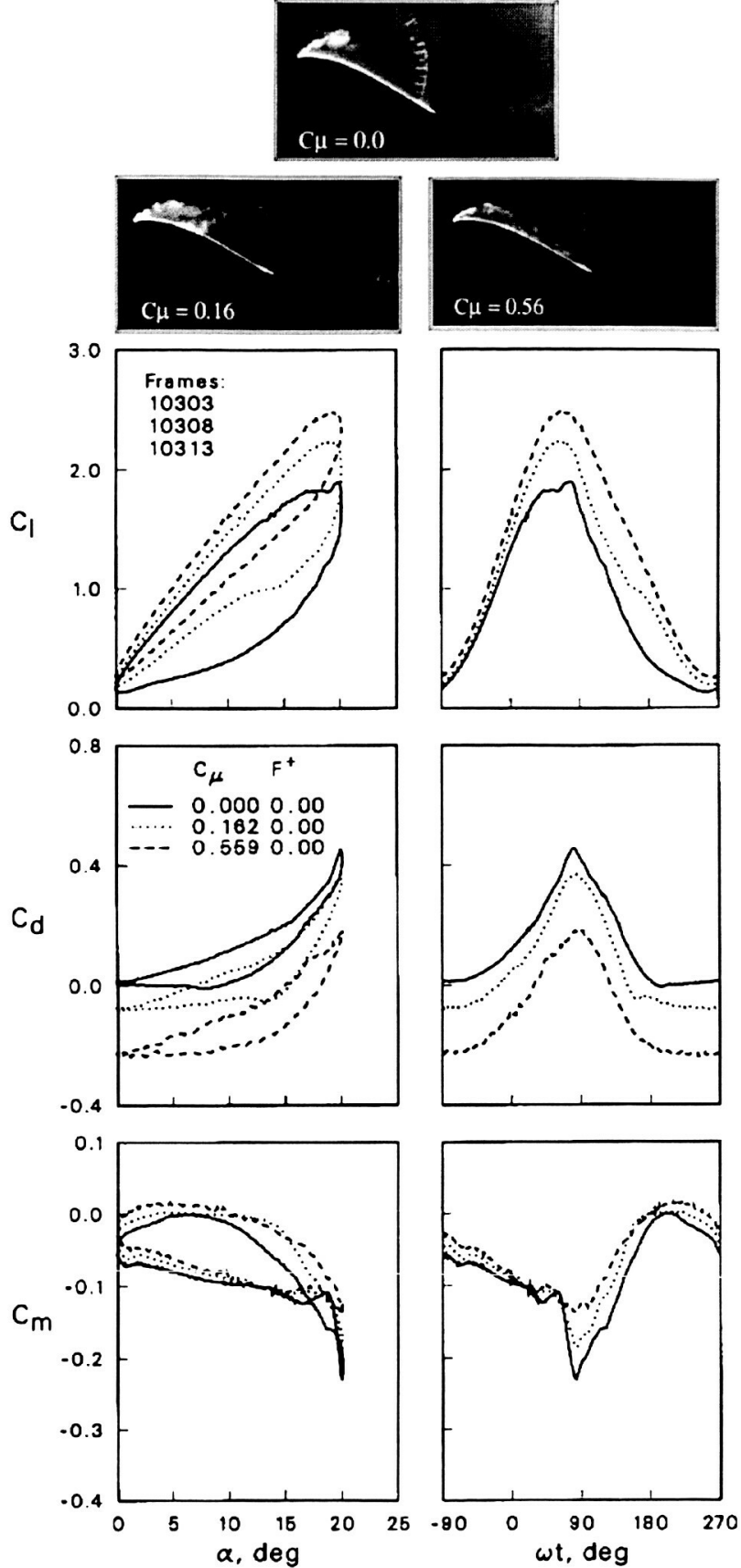

Fig. 6 Flow visualization (at $\alpha=20 \mathrm{deg}$ and $\omega t=90 \mathrm{deg}$ ) and load measurements for $\alpha_{m}=10 \mathrm{deg}$ and $k=0.15$ with different steady blowing rates.

moment stall). Blowing at both rates continues to yield a counterclockwise (positive damping) orientation throughout the moment cycle. Neither moment nor drag hysteresis is significantly reduced with blowing.

Flow visualizations ${ }^{17}$ show that deep stall occurs in the zeroblowing case, with reversed flow reaching the leading edge by $\omega t=40 \mathrm{deg}$. Formation of the dynamic stall vortex begins near $\omega t=45 \mathrm{deg}(\alpha=17.1 \mathrm{deg})$ and by $\omega t=70 \mathrm{deg}$ has moved over the surface to a location near $x / c=0.5$. By the time the airfoil has reached $\omega t=90 \mathrm{deg}$, the boundary layer has completely separated and is not reestablished until $\omega t=225 \mathrm{deg}(\alpha=2.9 \mathrm{deg})$. When the blowing rate is low, a separation bubble begins to form near $\boldsymbol{x} / \boldsymbol{c}=0.125$ at $\omega t=50 \mathrm{deg}$. At $\omega t=80 \mathrm{deg}$, the jet separates from the surface and a vortexlike structure is present near $\boldsymbol{x} / \boldsymbol{c}=0.5$ that appears to have been released when the bubble was 
compromised. No significant trailing-edge separation occurs in the high-blowing case. A separation bubble begins to form at approximately $\omega t=45 \mathrm{deg}$ and reaches its maximum size at $\omega t=70 \mathrm{deg}$, where it covers roughly $25 \%$ of the upper surface. Beyond this angle, the bubble appears to have opened up at the rear; however, the degree of leading-edge separation remains somewhat restrained. No vortexlike structures are observed at this blowing rate.

\section{Steady Blowing with $\alpha_{m}=15$ Degrees}

Although the mean angle has increased to $\alpha_{m}=15 \mathrm{deg}$, the quasi-steady sequence of boundary-layer events occurring between $\alpha=10$ and $20 \mathrm{deg}$ is essentially the same as that observed for $\alpha_{m}=10 \mathrm{deg}$. Beyond $20 \mathrm{deg}$, the boundary layer completely separates from the leading edge during each cycle. The general behavior
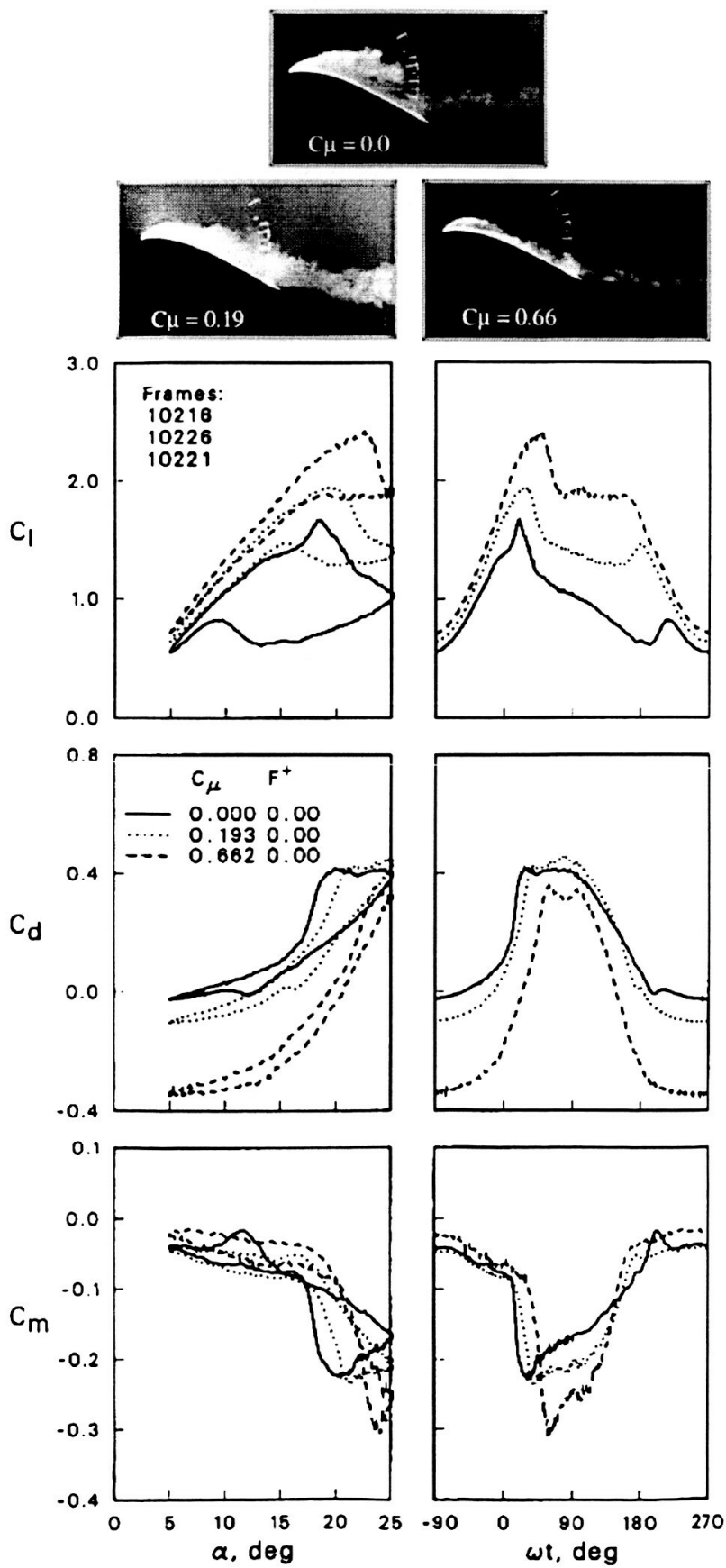

Fig. 7 Flow visualization (at $\alpha=18.4 \mathrm{deg}$ and $\omega t=20 \mathrm{deg}$ ) and load measurements for $\alpha_{m}=15 \mathrm{deg}$ and $k=0.05$ with different steady blowing rates. of the loads is virtually identical to the behavior at $\alpha_{m}=10 \mathrm{deg}$, the only difference being lower poststall values. No significant hysteresis is present during the quasi-steady oscillations with blowing.

Figure 7 shows a comparison of the results obtained with different blowing rates at $\alpha_{m}=15 \mathrm{deg}$ and $\boldsymbol{k}=0.05$. The effects of blowing are similar to those obtained at low amplitude $\left(\alpha_{m}=10 \mathrm{deg}\right)$ and summarized in Fig. 5. The primary differences involve the amount of hysteresis present and the maximum nose-down moment.

The increase in the magnitude of the pitching moment seen at the higher mean angle ( $\left.\alpha_{m}=15 \mathrm{deg}\right)$ is probably due to the formation of a much stronger vortexlike structure. In other words, as the blowing rate is increased, the separation bubble is trapped at the leading edge for a longer period of time. This allows the vorticity within the bubble to gain more strength. Whenever the blowing rate is
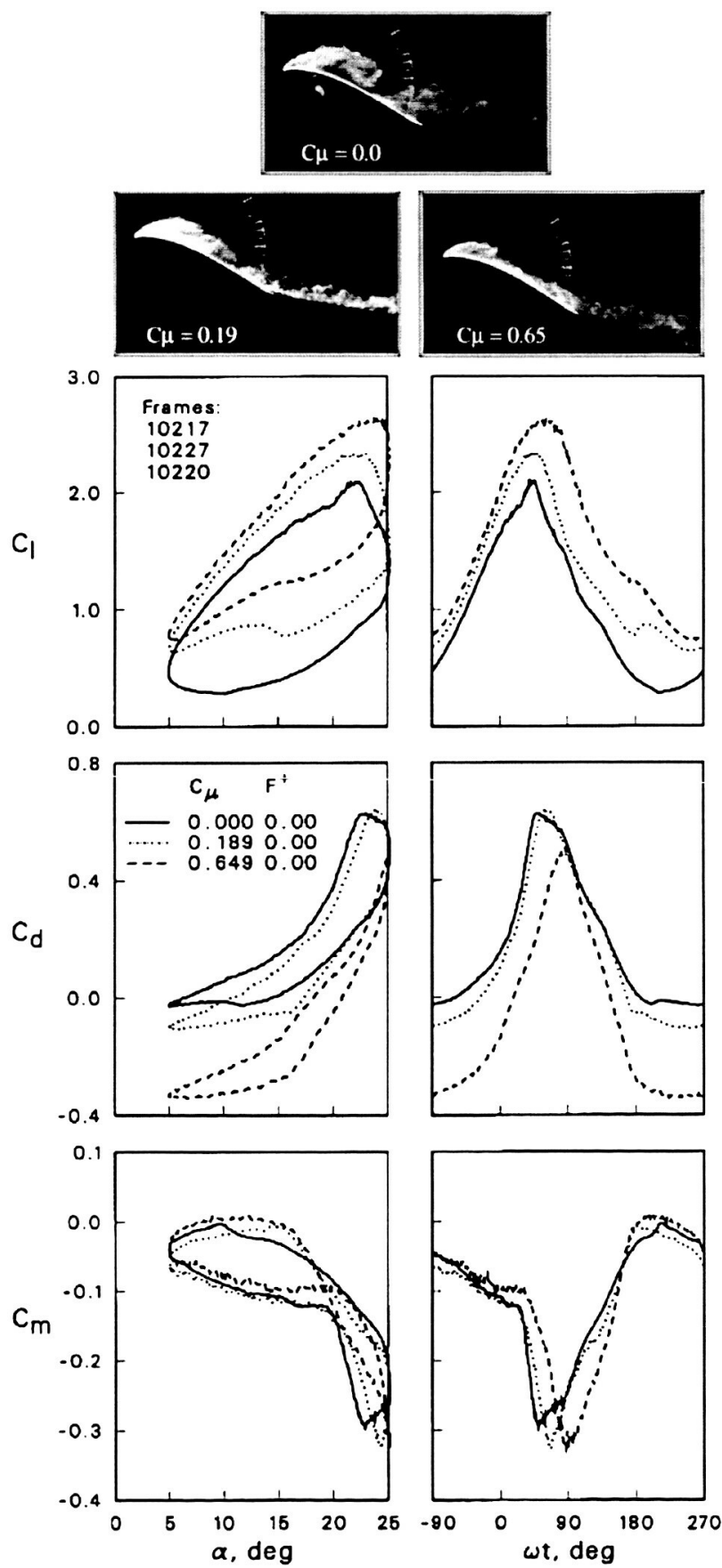

Fig. 8 Flow visualization (at $\alpha=22 \mathrm{deg}$ and $\omega t=45 \mathrm{deg}$ ) and load measurements for $\alpha_{m}=15 \mathrm{deg}$ and $k=0.15$ with different steady blowing rates. 
insufficient to maintain the bubble, a vortexlike structure is released, which migrates over the surface to produce a sizable change in the surface pressure distribution. A stronger vortex will alter the pressure distribution to a greater extent and cause a much larger nose-down moment as it passes off the surface. The larger vortex characterizes a significant change in the general flowfield, which requires a longer time to recover from. The increase in hysteresis that was observed is consistent with the larger disturbances present at these conditions.

By the increase of the reduced frequency to $k=0.15$, the airfoil experiences an even greater deep-stall penetration (Fig. 8). The trends are similar to those observed at the lower mean angle for the same reduced frequency (Fig. 6). Increasing the blowing rate progressively increases the lift curve slope during the upstroke, delays lift stall, increases the maximum-lift coefficient, and reduces
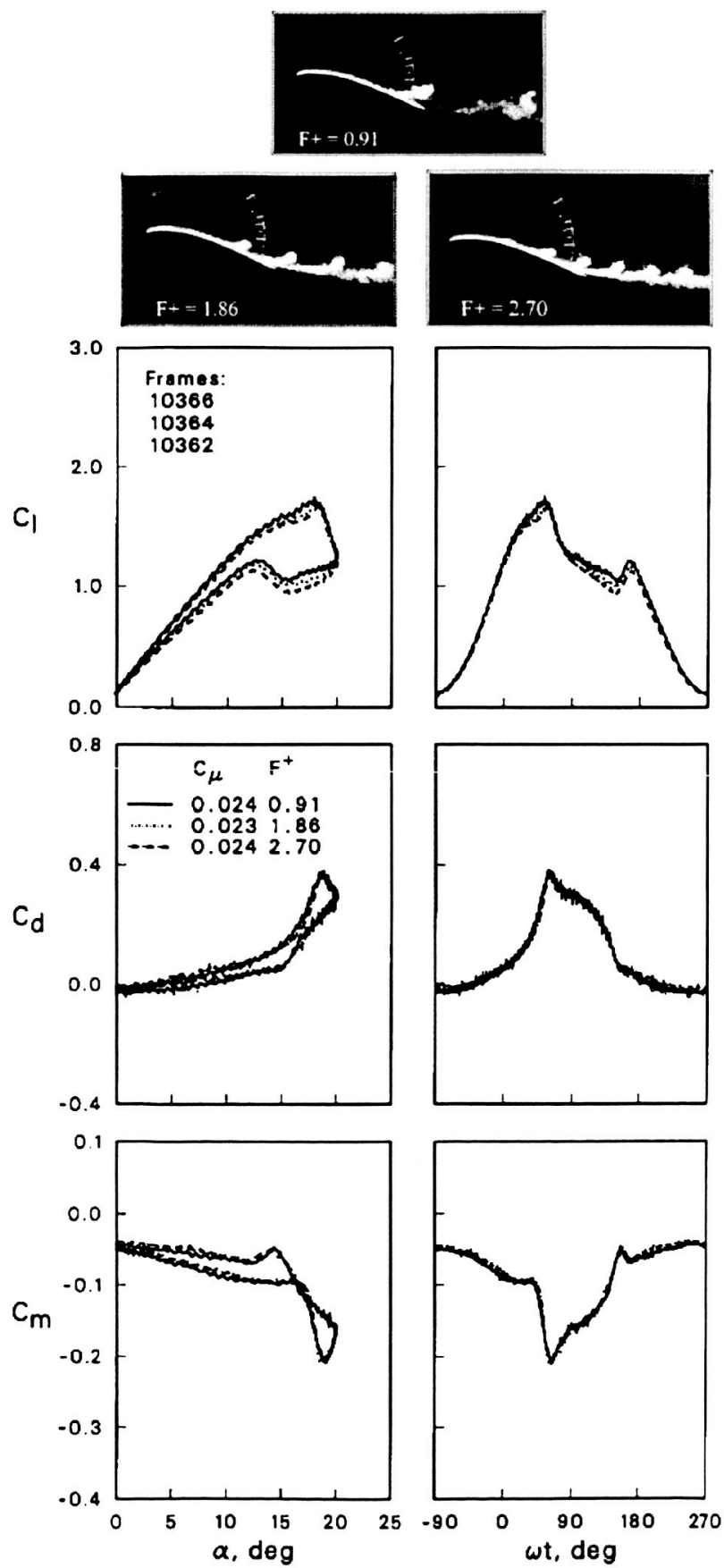

Fig. 9 Flow visualization (at $\alpha=13 \mathrm{deg}$ and $\omega t=20 \mathrm{deg}$ ) and load measurements for $\alpha_{m}=10 \mathrm{deg}$ and $k=0.05$ with different pulsed blowing rates. the lift hysteresis. Blowing also removes the distortion in the lift curve before stall. The primary difference between the high- and low-amplitude results is that, as the blowing rate is increased in the high-amplitude case, the peak-pitching moment is not reduced and a state of positive pitch damping is not always assured (possibly because of the limited $C_{\mu}$ available).

\section{Steady-Blowing Summary}

For each case studied, as $\boldsymbol{C}_{\boldsymbol{\mu}}$ increases, steady blowing not only increases the slope and maximum value of $C_{l}$, it also results in a large reduction in $\boldsymbol{C}_{d}$. The magnitude of the $\boldsymbol{C}_{d}$ reduction is as high as 0.09 for $\boldsymbol{C}_{\mu}=0.16$ and 0.24 for $\boldsymbol{C}_{\mu}=0.56$, which is an order of magnitude higher than the drag reduction with periodic excitation previously reported. ${ }^{19,20}$ Steady blowing also does not exhibit the drag penalty associated with the slatted and deformed airfoils. ${ }^{11-13}$
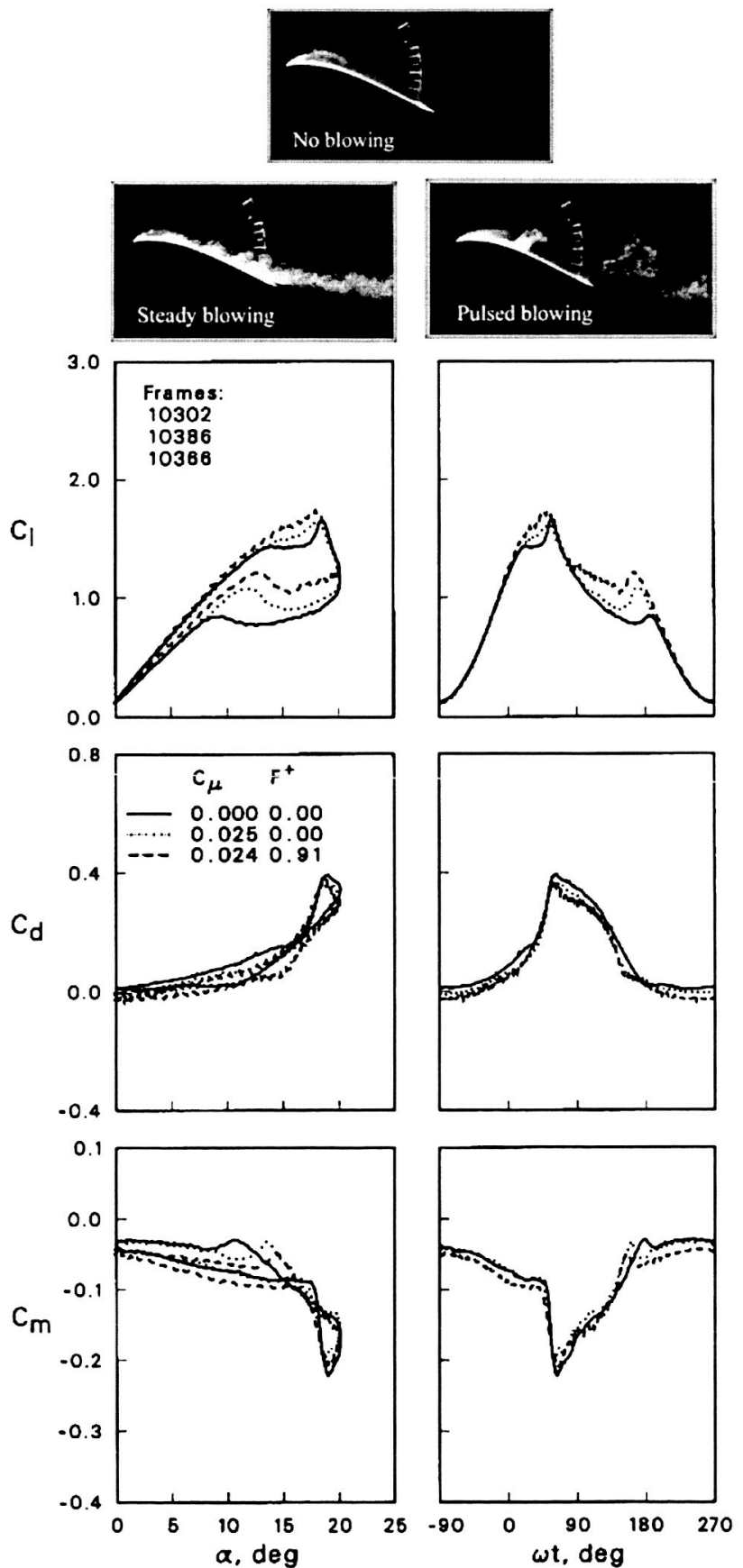

Fig. 10 Flow visualization (at $\alpha=16 \mathrm{deg}$ and $\omega t=40 \mathrm{deg}$ ) and load measurements for $\alpha_{m}=10 \mathrm{deg}$ and $k=0.05$ comparing no blowing with steady and pulsed blowing. 
This drag reduction can be attributed to the thrust effect of steady blowing and is roughly uniform over the entire angle range. The thrust should also yield minor gains in both the lift slope and $\boldsymbol{C}_{l, \max }$ These benefits are directly related to the blowing location, which in this experiment was at the quarter chord. Steady blowing at the leading edge would undoubtedly have a detrimental affect on the pitching moment and would not have a similar drag benefit due to jet thrust.

Steady blowing at the quarter-chord location appears to allow the formation of a separation bubble near the leading edge and inhibits the formation of a strong dynamic stall vortex. A further important consequence of steady blowing is the reshaping of the moment hysteresis to yield a positive pitch-damping condition. This is not necessarily the case for periodic excitation. ${ }^{19,20}$

\section{Pulsed Blowing at $\alpha_{m}=10$ Degrees and $\boldsymbol{k}=0.05$}

A few pulsed blowing cases were explored at a substantially reduced blowing rate. Results shown in Fig. 9 cover a range of $F^{+}=0-2.7$ for a constant momentum coefficient $\left(\boldsymbol{C}_{\mu}=0.024\right.$ nominal) and a fixed oscillation rate $(k=0.05$ nominal $)$ at $\alpha_{m}=10 \mathrm{deg}$. The curves are very similar to each other, indicating that there is only a marginal advantage to be gained from pulsed blowing at these conditions. However, pulsed blowing at $\boldsymbol{F}^{+}=0.9$ does yield a slightly better hysteresis reduction and a slightly higher increase in the maximum-lift coefficient.

In Fig. 10, the loads with no blowing are compared to the loads for steady blowing and pulsed blowing $\left(\boldsymbol{F}^{+}=0.9\right)$ at a blowing rate of $C_{\mu}=0.024$. The distortion in the lift curve during the upstroke, caused by the formation and movement of the dynamic stall vortex, is substantially smaller in the steady-blowing case and nearly disappears for pulsed blowing at $\boldsymbol{F}^{+}=0.9$. A slight increase in the maximum lift coefficient results from pulsed blowing. A reduction in the hysteresis occurs for each type of blowing, but pulsed blowing at $\boldsymbol{F}^{+}=0.9$ is the most effective. The boundary layer re-forms approximately 4 deg earlier with steady blowing and 5 deg earlier with pulsed blowing.

For similar low $\boldsymbol{C}_{\mu \mathrm{g}}$ onditions, pulsed blowing at the quarter chord provides a slight advantage in the lift behavior of the VR-7. Although not as obvious when the cycles are conditionally averaged, pulsed blowing creates substantial unsteadiness in the loads that is most pronounced in the poststall region. This is consistent with the numerical prediction for a NACA 0015 airfoil where a periodic excitation at the $10 \%$ chord location was found to cause a significant load oscillation at the excitation frequency. ${ }^{20}$ Pulsed blowing at $\boldsymbol{F}^{+}=0.9$ has a slight advantage in terms of hysteresis reduction and lift augmentation when compared to higher values of $\boldsymbol{F}^{+}$. For a NACA 0015 airfoil, the periodic excitation was found to be more effective than steady blowing with $C_{\mu}<1 \%$. When $C_{\mu}>2-3 \%$, steady blowing starts to become equal or better in effectiveness. ${ }^{19}$

\section{Conclusions}

A Boeing-Vertol VR-7 airfoil was experimentally studied with steady tangential upper-surface blowing at the quarterchord location for sinusoidal pitching oscillations described by $\alpha=\alpha_{m}+10$ deg $\sin \omega t$. The key conclusions of this study are as follows.

1) Steady blowing can significantly enhance the lift by trapping a separation bubble at the leading edge during the upstroke portion of the cycle. When a separation bubble can be trapped at the leading edge, moment stall and lift stall are eliminated, and the moment hysteresis loop is always in the direction of positive pitch damping.
2) Even when steady blowing is not strong enough to maintain the separation bubble for the entire cycle, a benefit is still realized through a substantial reduction of the lift hysteresis.

3) In addition to dynamic stall control, steady blowing results in a significant drag reduction due to jet thrust. This would not be the case for leading-edge blowing.

4) Although only a limited number of conditions were explored, pulsed blowing does not appear to deliver the performance improvements that have been successfully demonstrated in other studies involving zero-mass flow oscillatory blowing.

\section{References}

${ }^{1}$ McCroskey, W. J., McAlister, K. W., Carr, L. W., Pucci, S. L., Lambert, O., and Indergand, R. F., "Dynamic Stall on Advanced Airfoil Sections," Journal of the American Helicopter Society, Vol. 26, No. 3, 1981, pp. $40-50$.

${ }^{2}$ Prouty, R. W., Helicopter Performance, Stability, and Control, Prindle, Weber \& Schmidt, Boston, Massachusetts, 1986, Chap. 5.

${ }^{3} \mathrm{Ham}, \mathrm{N}$. D., and Young, M. I., "Torsional Oscillation of Helicopter Blades Due to Stall," Journal of Aircraft, Vol. 3, No. 3, 1966, pp. 218-224.

${ }^{4}$ Carr, L. W., McAlister, K. W., and McCroskey, W. J., "Analysis of the Development of Dynamic Stall Based on Oscillating Airfoil Experiments," NASA TN D-8382, Jan. 1977.

${ }^{5}$ McCroskey, W. J., "The Phenomenon of Dynamic Stall," NASA TM81264, March 1981.

${ }^{6} \mathrm{Ham}, \mathrm{N}$. D., "Aerodynamic Loading on a Two-Dimensional Airfoil During Dynamic Stall," AIAA Journal, Vol. 6, No. 10, 1968, pp. 1927-1934.

${ }^{7}$ Van Dommelen, L., and Shen, S. F., "The Spontaneous Generation of the Singularity in a Separating Laminar Boundary Layer," Journal of Computational Physics, Vol. 38, No. 2, 1980, pp. 125-140.

${ }^{8}$ Choudhuri, P. G., Knight, D. D., and Visbal, M. R., "Two-Dimensional Unsteady Leading-Edge Separation on a Pitching Airfoil," AIAA Journal, Vol. 32, No. 4, 1994, pp. 673-681.

${ }^{9}$ Shih, C., Lourenco, L. M., and Krothapalli, A., "Investigation of Flow at Leading and Trailing Edges of Pitching-Up Airfoil," AIAA Journal, Vol. 33, No. 8, 1995, pp. 1369-1376.

${ }^{10}$ Walker, J. D. A., "Unsteady Boundary Layer Separation in TwoDimensional Flows," Proceedings of the AFOSR Workshop on Supermaneuverability, Physics of Separated Flows at High Angle of Attack, Lehigh Univ., Bethlehem, PA, April 1992.

${ }^{11}$ McAlister, K. W., and Tung, C., "Suppression of Dynamic Stall with a Leading-Edge Slat on a VR-7 Airfoil," NASA TP-3357, March 1993.

${ }^{12}$ Plantin de Hugues, P., McAlister, K. W., and Tung, C., "Effect of an Extendable Slat on the Stall Behavior of a VR-12 Airfoil," NASA TP-3407, Sent. 1993.

${ }^{13}$ Lee, S., McAlister, K. W., and Tung, C., "Characteristics of a Deformable Leading Edge for High Performance Helicopter Rotor," AIAA Paper 93-3526, Aug. 1993.

${ }^{14}$ Sahin, M., Sankar, L. N., Chandrasekhara, M. S., and Tung, C., "Dynamic Stall Alleviation Using a Deformable Leading Edge Concept: A Numerical Study," Journal of Aircraft, Vol. 40, No. 1, 2003, pp. 77-85.

${ }^{15}$ Chanderasekhera, M. S., Wilder, M. C., and Carr, L. W., "Compressible Dynamic Stall Control Using Dynamic Shape Adaption," AIAA Journal, Vol. 39, No. 10, 2001, pp. 2021-2024.

${ }^{16}$ Yu, Y. H., Lee, S., McAlister, K. W., Tung, C., and Wang, C. M., "High Lift Concepts for Rotorcraft Applications," The 49th American Helicopter Society Annual Forum, May 1993, pp. 1141-1166.

${ }^{17}$ Weaver, D., McAlister, K. W., and Tso, J., "Suppression of Dynamic Stall by Steady and Pulsed Upper-Surface Blowing," AIAA Paper 98-2413, June 1998.

${ }^{18}$ Greenblatt, D., and Wygnanski, I., "Parameters Affecting Dynamic Stall Control by Oscillatory Excitation," AIAA Paper 99-3121, June 1999.

${ }^{19}$ Greenblatt, D., and Wygnanski, I., "Dynamic Stall Control by Periodic Excitation, Part I, NACA 0015 Parametric Study," Journal of Aircraft, Vol. 38, No. 3, 2001, pp. 430-438.

${ }^{20}$ Ekaterinaris, J. A., "Numerical Investigation of Dynamic Stall Active Control for Incompressible and Compressible Flows," Journal of Aircraft, Vol. 39, No. 1, 2002, pp. 71-78. 